\title{
Correction: Re-examining the link between childhood maltreatment and substance use disorder: a prospective, genetically informative study
}

Andrea J. Capusan • Per A. Gustafsson • Ralf Kuja-Halkola (D) Kajsa Igelström • Leah M. Mayo (D) Markus Heilig (D)

Published online: 30 April 2021

(c) The Author(s), under exclusive licence to Springer Nature Limited 2021

Correction to: Molecular Psychiatry

https://doi.org/10.1038/s41380-021-01071-8
The original version of this article contained a mistake in the spelling of Leah M. Mayo's name. The original article has been corrected. 